\title{
High frequency of copy number imbalances in Rubinstein-Taybi patients negative to CREBBP mutational analysis
}

\author{
Cristina Gervasini $^{1,8}$, Federica Mottadelli ${ }^{1,8}$, Roberto Ciccone $^{2}$, Paola Castronovo ${ }^{1}$, Donatella Milani ${ }^{3}$, \\ Gioacchino Scarano ${ }^{4}$, Maria Francesca Bedeschi ${ }^{5}$, Serena Belli ${ }^{6}$, Alba Pilotta ${ }^{7}$, Angelo Selicorni ${ }^{3}$, \\ Orsetta Zuffardi $^{2}$ and Lidia Larizza ${ }^{\star, 1}$
}

Rubinstein-Taybi syndrome (RSTS) is a rare autosomal dominant disorder characterised by facial dysmorphisms, growth and psychomotor development delay, and skeletal defects. The known genetic causes are point mutations or deletions of the CREBBP (50-60\%) and EP3OO (5\%) genes. To detect chromosomal rearrangements indicating novel positional candidate RSTS genes, we used a-CGH to study 26 patients fulfilling the diagnostic criteria for RSTS who were negative at fluorescence in situ hybridisation analyses of the CREBBP and EP300 regions, and direct sequencing analyses of the CREBBP gene. We found seven imbalances (27\%): four de novo and three inherited rearrangements not reported among the copy number variants. A de novo 7p21.1 deletion of $500 \mathrm{~kb}$ included the TWIST1 gene, a suggested candidate for RSTS that is responsible for the Saethre-Chotzen syndrome, an entity that enters in differential diagnosis with RSTS. A similar issue of differential diagnosis was raised by a large $4.3 \mathrm{Mb} 2 \mathrm{q} 22.3 \mathrm{q} 23.1$ deletion encompassing ZEB2, the gene responsible for the Mowat-Wilson syndrome, whose signs may overlap with RSTS. Positional candidate genes could not be sought in the remaining pathogenetic imbalances, because of the size of the involved region (a $9 \mathrm{Mb} 2 q 24.3 q 31.1$ deletion) and/or the relative paucity of suitable genes (a $5 \mathrm{Mb}$ $3 p 13 p 12.3$ duplication). One of the inherited rearrangements, the $17 q 11.2379 \mathrm{~Kb}$ duplication, represents the reciprocal event of the deletion underlying an overgrowth syndrome, both being mediated by the NF1-REP-P1 and REP-P2 sub-duplicons. The contribution of this and the other detected CNVs to the clinical RSTS phenotype is difficult to assess.

European Journal of Human Genetics (2010) 18, 768-775; doi:10.1038/ejhg.2010.1; published online 3 February 2010

Keywords: Rubinstein-Taybi syndrome; array-CGH; genomic imbalances; differential diagnosis; genotype-phenotype correlations

\section{INTRODUCTION}

Rubinstein-Taybi syndrome (RSTS, MIM \#180849) is an autosomal dominant disease that occurs in 1 out of 125000 births. Affected patients are characterised by growth and psychomotor development delay because of the major involvement of the skeletal and central nervous systems. The main skeletal features are radially diverted phalanges (in one-third of the cases) and broad and duplicated distal phalanges of the thumbs and halluces (99\% of the patients), which are hallmark signs of the syndrome. Typical facial dysmorphisms such as down-slanting palpebral fissures and a prominent beaked nose help clinical geneticists make a diagnosis. ${ }^{1}$ The patients are also at increased risk of tumours, ${ }^{2}$ especially those involving the epidermis such as pilomatrixomas ${ }^{3}$ and epidermal nevi. ${ }^{4}$

RSTS is caused by mutations in two genes: cAMP response elementbinding protein (CREBBP, also known as ' $C B P$ ') localised at $16 \mathrm{p} 13.3,{ }^{5}$ and E1A-associated protein p300 (EP300), localised at 22q13. Both genes encode histone acetyltransferases (HATs), transcriptional co-activators that are involved in cell processes such as growth, differentiation, DNA repair, apoptosis and many others, ${ }^{7}$ and which also have an important role in the development of the skeletal and nervous central systems, thus accounting for the growth and psychomotor development delay typical of RSTS patients. Various techniques have been used to identify the genetic lesion underlying RSTS, including fluorescence in situ hybridisation (FISH), ${ }^{8-11}$ real-time quantitative $\mathrm{PCR},{ }^{12}$ multiplex ligation-dependent probe amplification (MLPA), ${ }^{13}$ denaturing high performance liquid chromatography (DHPLC) ${ }^{14}$ and sequencing. ${ }^{15,16}$ Deletions of part or all of the CREBBP gene and flanking regions (also in mosaic condition), ${ }^{11}$ may account for $5-10 \%$ of the cases, whereas frameshift, nonsense, splice-site and missense point mutations (in decreasing order of prevalence) are found in approximately $46-51 \% ;{ }^{17}$ there have also been reports of a few gene-disrupting translocations and inversions. $^{10}$

EP300 mutations are comparatively rare as only six have been described so far, in six patients with a mild clinical presentation. ${ }^{6,18-20}$ The pathological mechanism has been clarified in approximately $60 \%$

${ }^{1}$ Division of Medical Genetics, San Paolo School of Medicine, University of Milan, Milan, Italy; ${ }^{2}$ Sezione di Biologia Generale e Genetica Medica, Università di Pavia, Italy; ${ }^{3}$ Clinica Pediatrica, Fondazione Policlinico Mangiagalli Regina Elena, Milan, Italy; ${ }^{4}$ UO Genetica Medica, AO 'G Rummo', Benevento, Italy; ${ }^{5}$ Clinical Genetic Unit, Fondazione Ospedale Maggiore Policlinico Mangiagalli e Regina Elena, Department of Obstetrics and Pediatrics, Milan, Italy; ${ }^{6}$ Consultorio Genetico APSS, Trento, Italy; ${ }^{7}$ Auxoendocrinologia, Ospedale Pediatrico, Brescia, Italy

*Correspondence: Dr L Larizza, Division of Medical Genetics, San Paolo School of Medicine, University of Milan, Milan, Italy Via A.di Rudinì, 8 20142 Milan, Italy. Tel: +39 025032 3206; Fax: +3902 5032 3026; E-mail: lidia.larizza@unimi.it

${ }^{8}$ These authors contributed equally to this work.

Received 7 May 2009; revised 8 September 2009; accepted 9 December 2009; published online 3 February 2010 
of the cases, but multiple cryptic mutational mechanisms of the major CBP gene may be overlooked, including mosaic point mutations, mutations affecting the $5^{\prime}$ and $3^{\prime}$ UTRs, promoter epimutations and alterations at post- transcriptional or translational levels.

In brief, RSTS is a heterogeneous disorder because of at least two different genes, thus allowing speculation that other genes may also be involved and lead to the observed spectrum of clinical presentations.

Array comparative genomic hybridisation (a-CGH) is a powerful means of interrogating the overall genome for copy number alterations, but has so far only been used to analyse the CREBBP region in RSTS patients. ${ }^{21}$ The aim of this genome-wide assay was to identify rearrangements that might underlie RSTS-like phenotypes, and possibly detect positional candidate genes, in 26 Italian patients with a clinical diagnosis of RSTS who were negative at FISH analyses of the $C R E B B P$ and $E P 300$ regions and sequencing analyses of the CREBBP gene. We found seven imbalances (four de novo and three inherited rearrangements) and discuss their implications in RSTS and in related syndromic entities.

\section{MATERIALS AND METHODS}

The 26 patients analysed by means of array CGH had a clinical diagnosis of RSTS, and were negative at FISH analyses of the CREBBP and EP300 regions, and direct sequencing analyses of the CREBBP gene. Genomic DNA was isolated from the patients' peripheral blood samples using a QIAamp DNA Blood Kit (Qiagen, Hilden, Germany).

\section{Array CGH analysis}

All of the patients were analysed using the $4 \times 44 \mathrm{~K}$ genome-wide chip (Agilent Technologies, Santa Clara, CA, USA), which has an average resolution of $100 \mathrm{~kb}$. The parents of the carriers of genomic imbalances were also analysed.

Aliquots of $500 \mathrm{ng}$ of patient and reference DNAs (Promega Corporation, Madison, WI, USA) were double-digested with RsaI and AluI (Promega) for $2 \mathrm{~h}$ at $37^{\circ} \mathrm{C}$. After heat inactivation of restriction endonucleases at $65^{\circ} \mathrm{C}$ for $20 \mathrm{~min}$, each digested sample was labelled by means of the Genomic DNA Labelling Kit PLUS (Agilent Technologies) for $2 \mathrm{~h}$ at $37^{\circ} \mathrm{C}$, using Cy5-dUTP for the patient DNAs and Cy3-dUTP for the reference DNAs. After purification from non-incorporated fluorochromes, the DNAs were properly combined and denatured for $3 \mathrm{~min}$ at $95^{\circ} \mathrm{C}$. After $30 \mathrm{~min}$ pre-annealing with $5 \mu \mathrm{g}$ of Cot- 1 DNA, the samples were hybridised at $65^{\circ} \mathrm{C}$ for $24 \mathrm{~h}$ with rotation, and then the non-hybridised DNA was removed by means of two post-hybridisation washings. Images of the chips were acquired using the Agilent scanner and analysed by means of Feature Extraction 9.5 software 9.5 (Agilent); the results were graphically represented using CGH Analytics 3.5 software (Agilent).

\section{Fluorescence in situ hybridisation}

The chromosome preparations were obtained using standard cytogenetic techniques. Briefly, phytohemagglutinin(PHA)-stimulated peripheral blood lymphocytes were set up in culture using the 'Synchro' Chromosome Kit
(Celbio, Milan, Italy) and modified RPMI (Irvine Scientific, Santa Ana, CA, USA) plus 5\% fetal calf serum (Gibco LTD, Paisley, Scotland). The cultures were stopped with colchicine after $72 \mathrm{~h}$.

The CREBBP and EP300 encompassing BAC probes were selected on the basis of their physical location (http://www.genome.ucsc.edu/): details are reported in Gervasini et al, 2007. DNA was isolated from liquid cultures using a plasmid purification kit (Nucleobond PC20, Macherey-Nagel, GmbH \& Co.KG, Duren, Germany). The BAC clones were labelled with digoxigenin11-dUTP or biotin-16-dUTP (Roche Diagnostics, Mannheim, Germany) using a nick translation kit (Roche Diagnostics). The FISH experiments were performed using standard procedures. ${ }^{22}$ The chromosomes were counterstained with DAPI in antifade Vectashield Mounting Medium (Vector Laboratories Inc., Burlingame, CA, USA), and visualised using a Leitz DM-RB microscope (Leica Microsystems GmbH, Wetzlar, Germany) equipped for DAPI and FITC/TRITC epifluorescence optics. The images were captured by means of a CCD camera High Performance CCD Camera (COHU, Poway, CA, USA), and visualised using McProbe software (Applied Imaging, PowerGene, League City, TX, USA).

\section{Microsatellite segregation analysis}

Segregation from parents to probands was analysed using the D2S2188, D2S399, D3S3653 and D3S4533 fluorescent dye-labelled microsatellite markers, with the fluorescence being detected by means of an ABI 3100 sequencer. ABI PRISM software Genescan (Applied Biosystem, Foster City, CA, USA) was used for gel analysis.

\section{SNP sequence analysis}

The DNA direct sequencing of the SNPs rs2084674, rs 34729008 and rs2084674 was performed by means of an ABI PRISM 3130 sequencer (Applied Biosystem), and the electropherograms were analysed using Chromas Pro version 1.42 software (Technelysium Pty LTD, Australia).

\section{RESULTS}

\section{Overview of array CGH findings}

The 26 RSTS patients ( 14 men and 12 women aged between 6 months and 42 years) had clinical diagnoses ranging from definite (consistent) to probable (suggestive and within the spectrum) or possible (borderline or RSTS-like). This cohort was selected because the patients were negative for CREBBP point mutations and chromosomal rearrangements affecting the CREBBP and EP300 regions, and thus consistent with our main aims of detecting genomic regions with sequence gains or losses (to be analysed in further detail in order to refine the clinical diagnosis) and identifying novel candidate RSTS genes. Seven of the 26 patients carried DNA copy number alterations, including four deletions and three duplications: the de novo imbalances were two large deletions of chromosome $2 \mathrm{q}$ (one encompassing $9 \mathrm{Mb}$ at $2 \mathrm{q} 24.3 \mathrm{q} 31.1$ and the other $4.3 \mathrm{Mb}$ at 2q22.3q23.1), a small deletion of chromosome 7p21.1, and a $5 \mathrm{Mb}$ duplication at 3p13p12.3 (Table 1). Table 2 shows the clinical signs of these four cases, and Figure 1 shows the facial appearance and typical right thumb/hallux of three of them.

Table 1 De novo and inherited CNV detected by means of array-CGH in a set of 26 CREBBP-negative RSTS patients

\begin{tabular}{|c|c|c|c|c|c|}
\hline Clinical RSTS diagnosis & Patient & Copy number variation & Size $(M b)$ & Boundaries $(b p)^{a}$ & Origin \\
\hline Probable & 51 & $\operatorname{Del}(2)(q 22.3 q 23.1)$ & 4.3 & $144714856-149002435$ & De novo (paternal) \\
\hline Definite & 14 & $\operatorname{Del}(2)(q 24.3 q 31.1)$ & 9.1 & $167905353-176958852$ & De novo (paternal) \\
\hline Definite & 61 & Dup (3)(p13p12.3) & 5.1 & 73 108649-78239462 & De novo (maternal) \\
\hline Possible & 58 & $\operatorname{Del}(7)(\mathrm{p} 21.1)$ & 0.5 & $18868958-19335351$ & De novo $(-)$ \\
\hline Definite & 45 & Dup (2)(q34q35) & 4.9 & $212860355-217724468$ & Inherited (paternal) ${ }^{\mathrm{b}}$ \\
\hline Definite & 29 & Dup (17)(q11.2) & 0.4 & $28941066-29320612$ & Inherited (paternal) ${ }^{\mathrm{b}}$ \\
\hline Definite & 78 & $\operatorname{Del}(18)(q 22.1)$ & 1.3 & $64866077-66190392$ & Inherited (paternal) ${ }^{\mathrm{b}}$ \\
\hline
\end{tabular}


Table 2 RSTS spectrum and patient-specific clinical signs of the four patients carrying de novo imbalances

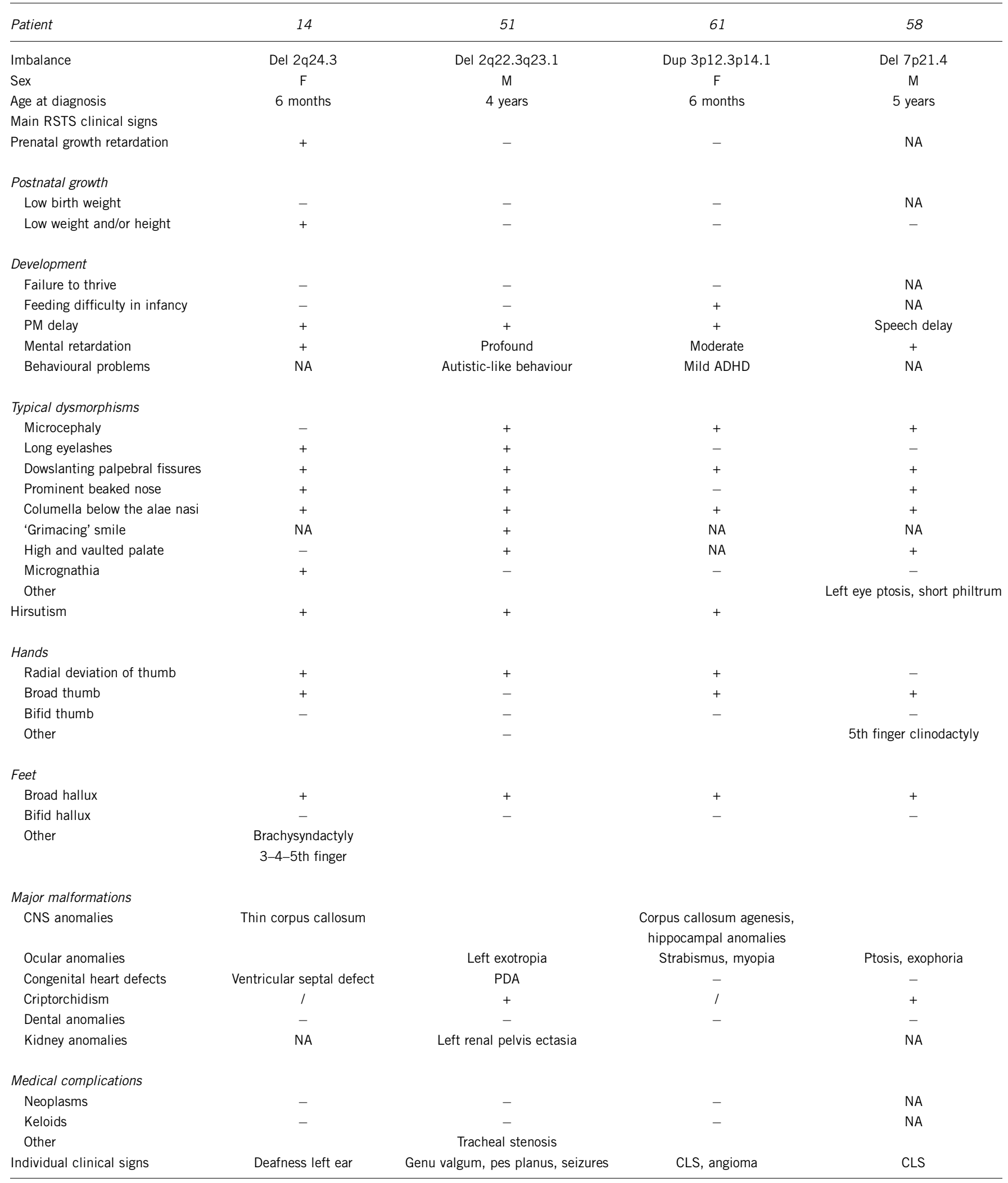

Abbreviations: ADHD, attention-deficit hyperactivity disorder; CLS, café au lait spots; PDA, patent ductus arteriosus; NA, not assessed. 
a
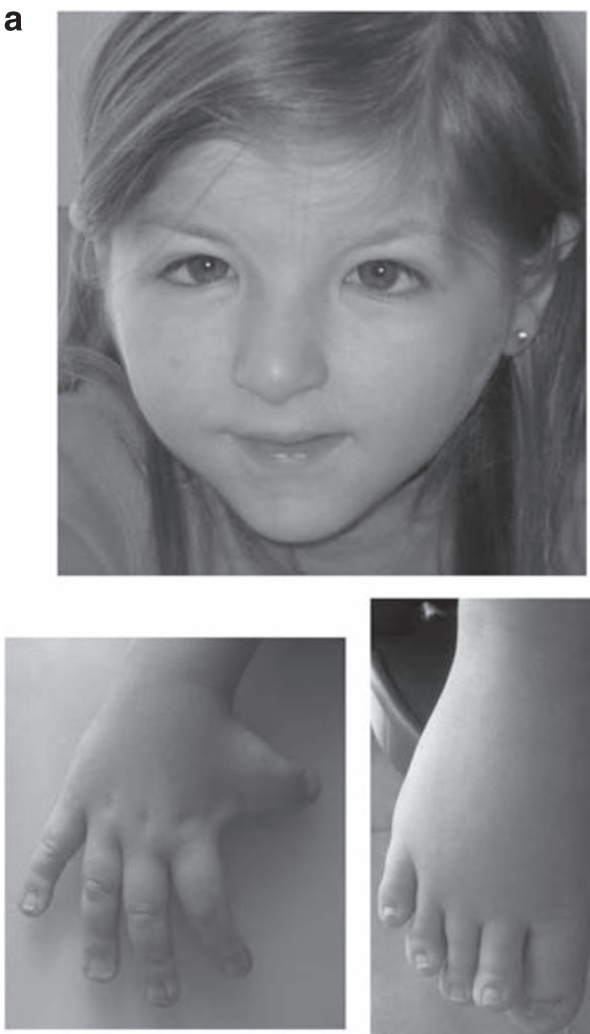

b
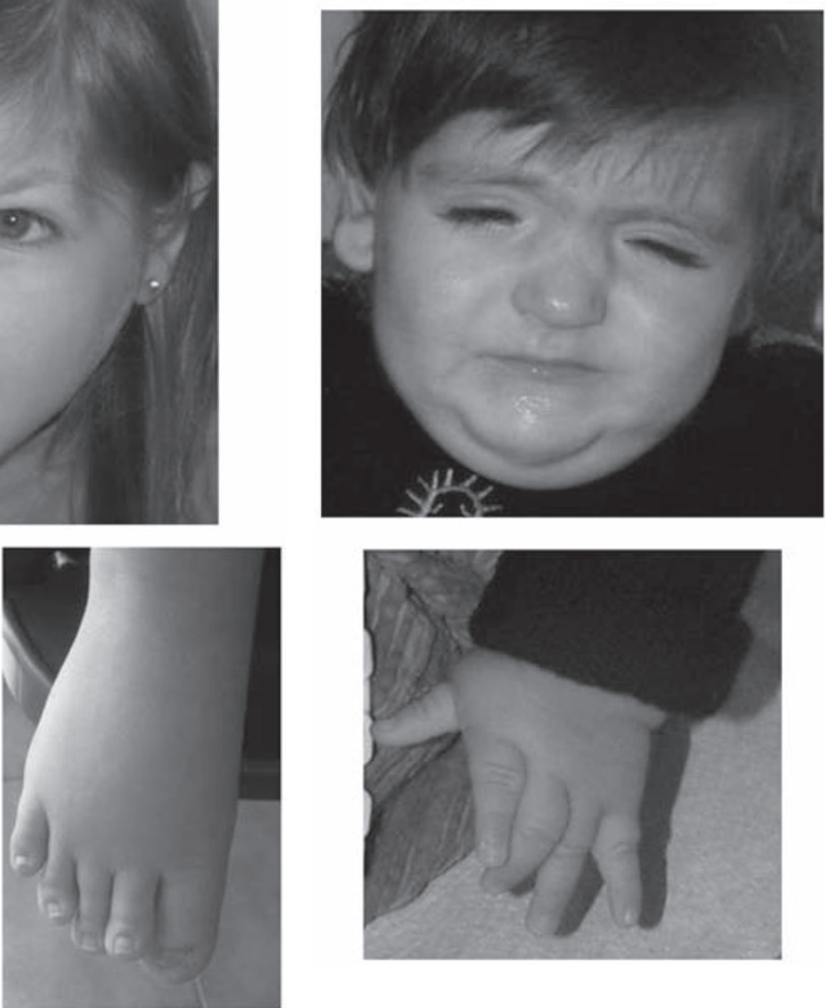
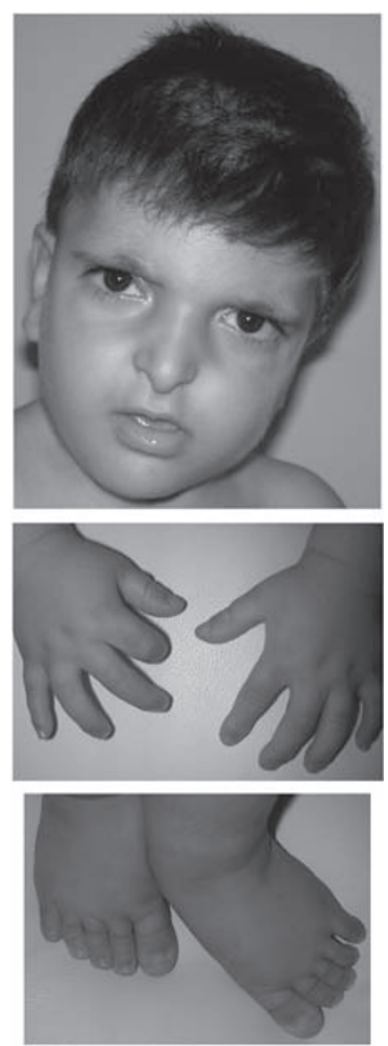

Figure 1 Facial features and hand-foot anomalies in RSTS patients carrying de novo imbalances: patient 61 (a), patient 14 (b) and patient 51 (c).

Details regarding the genomic regions involved in the de novo/ inherited imbalances

Patient 51. The $4.3 \mathrm{Mb} 2 \mathrm{q} 22.3 \mathrm{q} 23.1$ deletion was confirmed by FISH using the CTD2162B21 BAC clone. Segregation analysis of SNPs rs2084674 (and rs34729008 mapping within the deletion) showed that the deletion arose de novo on the paternally contributed chromosome as independently confirmed by array CGH of the parents' DNA (data not shown). Six RefSeq genes are located in the deleted interval, including the zinc finger homeobox 1B ZEB2 that is responsible for Mowat-Wilson syndrome (MWS) (OMIM\#235730) (Figure 2a).

Patient 14. The 9Mb 2q24.3q31.1 deletion involves $>50$ RefSeq genes and the HOXD gene cluster in its distal HOXD13 element (Figure $2 \mathrm{~b}$ ). The distal breakpoint was validated and characterised by means of double-colour metaphase FISH using the CTD-2226C5 (specific for the whole HOXD cluster) and RP11-892L20 BAC (covering the upstream regulatory region), which respectively map outside and inside the deletion. The same BAC array-CGH and FISH analyses were applied to both parents and confirmed the de novo origin of the deletion. Segregation analysis using microsatellites D2S2188 and D2S399 (both mapping within the deletion) showed that the deletion arose de novo on the paternally contributed chromosome (data not shown). Using the RP11-471A5 and RP11892L20 BACs (mapping inside the deletion) and CTD-2226B10 (mapping outside) to study the metaphases/nuclei of the father's chromosomes allowed us to exclude the presence of an inversion predisposing the region to break (data not shown). Previous karyotyping also revealed the presence in the patient of a marker chromosome (data not shown) that was not characterised in detail. The genes localised within the deleted region that are worth noting include: (i) HOXD13, which encodes a transcription factor that has an important role in morphogenesis; (ii) the $D L X 1$ and $D L X 2$ genes encoding proteins that may have a role in controlling craniofacial patterning, as well as in the differentiation and survival of inhibitory neurons in the forebrain; and (iii) the gene for activating transcription factor 2 (ATF2, also called CREBP1 or CREB2) whose product stimulates the CRE (cAMPresponsive element)-dependent genes and has HAT activity specific for histones $\mathrm{H} 2 \mathrm{~B}$ and $\mathrm{H} 4$.

Patient 61. The 3p13p12.3 duplication spans $5.1 \mathrm{Mb}$ and harbours seven RefSeq genes (Figure 2c). Segregation analysis using microsatellites D3S3653 and D3S4533 (mapping within the large duplicated segment) showed its de novo origin on the maternally contributed chromosome, in line with the 'normal' array CGH profile of both parents' DNA. The duplicated genes of possible pathogenetic relevance are the roundabout, axon guidance receptor, homologue 2 (ROBO2) gene, which encodes a protein receptor for SLIT Drosophila homologue 2 (SLIT2) that is known to function in axon guidance and cell migration, and the contactin 3 (CNTN3), a member of the contactin family that mediates cell surface interactions during nervous system development.

Patient 58. The small deletion of $466 \mathrm{~kb}$ in 7 p21.1 (Figure $2 \mathrm{~d}$ ) affects the region containing TWIST1, the causative gene of Saethre-Chotzen syndrome (SCS) (OMIM\#101400) and interrupts HDAC9, a gene coding for a protein whose function is the opposite of that of CREBBP. The deletion was validated by FISH analysis using BAC clone CTD-2050C8, and its de novo origin was confirmed by parent's analysis.

Patients 45, 78 and 29. The deletion of patient 78 (which spans $1.2 \mathrm{Mb}$ in $18 \mathrm{q} 22.1$ ) and the $5.5 \mathrm{Mb} 2 \mathrm{q} 34 \mathrm{q} 35$ duplication of patient 45 , 

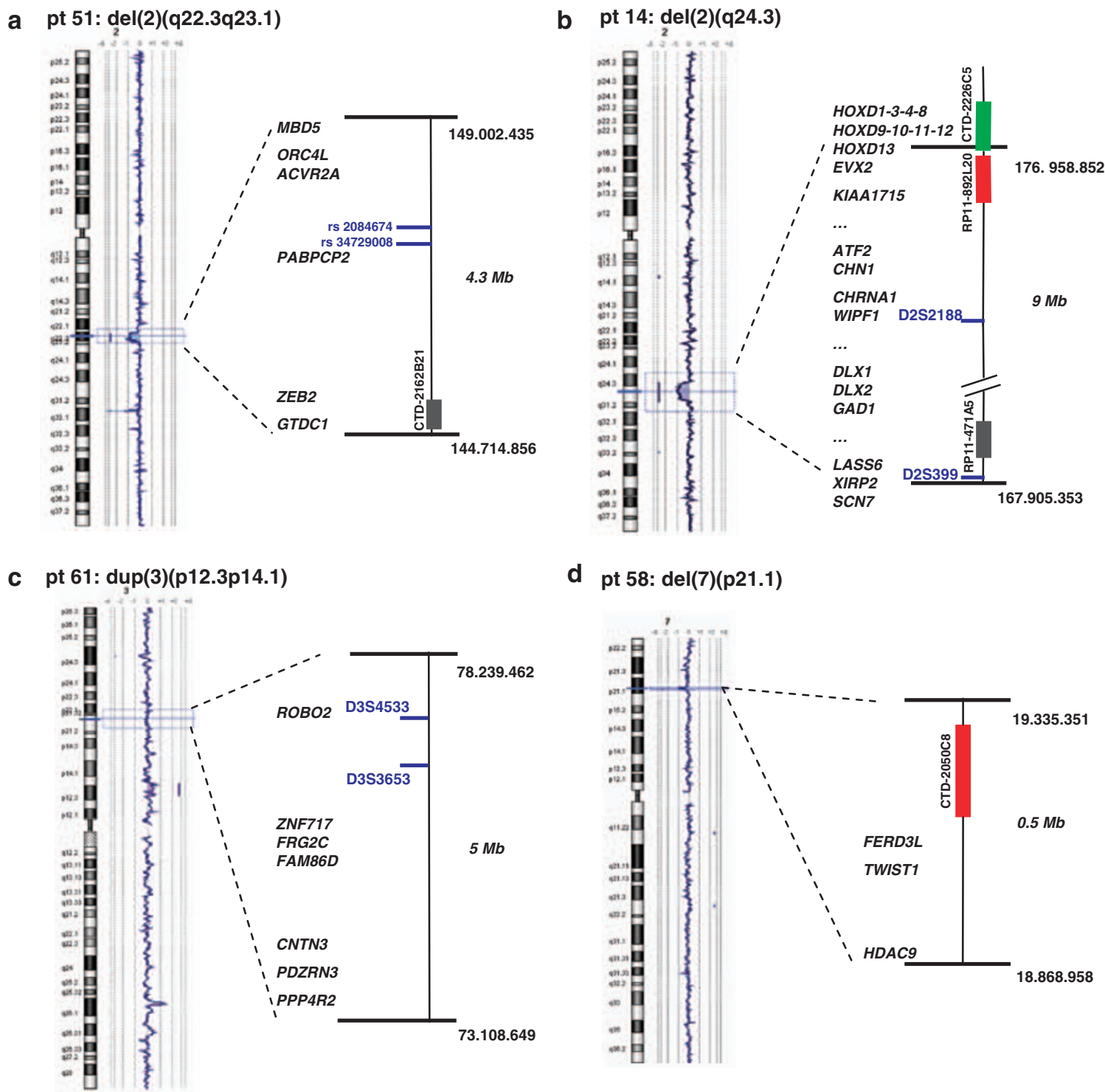

d pt 58: del(7)(p21.1)

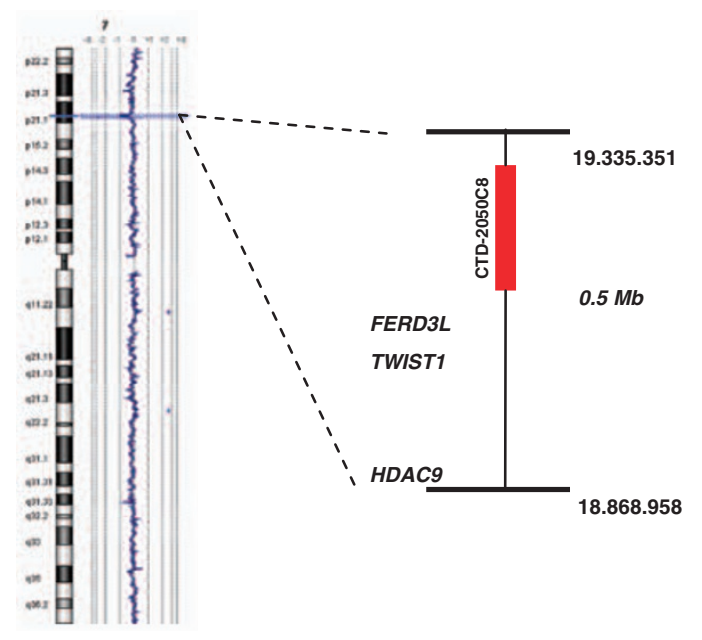

Figure 2 Mapping of the four identified de novo imbalances with the chromosome ideogram (left) and the corresponding aCGH profile: on the right, the region involved in the deletion/duplication is zoomed to show the genes possibly involved in the clinical phenotype and the markers (BACs/SNPs/ microsatellites) used for the validation. The breakpoints are indicated by black horizontal bars according to the UCSC Genome Browser on Human, February 2009 Assembly.

shown by array CGH to be also carried by the patients' fathers, involve gene-poor regions. A bioinformatic search (Human Genome Segmental Duplication Database http://projects.tcag.ca/humandup/) revealed that these regions are not numbered copy number polymorphisms.

The duplication carried by patient 29 (Figure 3 ) only affects $379 \mathrm{~kb}$ of the 17q11.2 region upstream of the NF1 gene, which is delimited by the sub-duplicons REP-P1/P2 of the proximal NF1-REP P (Figure 3c). The duplication was confirmed by FISH analysis using BAC clone RP11-753N3 and excluded in the healthy mother (data not shown). Although the father was unavailable for testing, his carrier status could be inferred from the array $\mathrm{CGH}$-assessed presence of the same duplication in the healthy patient's sister.

\section{DISCUSSION}

RSTS is a genetically heterogeneous disorder with a causative genetic lesion only detected in $50-60 \%$ of cases. ${ }^{15,16,23}$ This low mutation rate may be attributable to overlooked mosaic point mutations, incomplete coverage in the sequencing procedure of the regulatory and intronic regions of the known genes (CREBBP and EP300), or the involvement of additional RSTS-causing genes.

We addressed this last possibility using array CGH to process a cohort of patients with a definite, probable or possible clinical diagnosis of RSTS, who were negative to mutation/deletion scans of $C R E B B P$ and the FISH analysis of EP300, and found that 7 out of 26 patients (27\%) bore genomic imbalances. The rearrangements were de novo in four cases, and were therefore assessed as possible causes of the phenotype. A reassessment of the carriers of the de novo imbalances confirmed the presence of the main clinical signs of RSTS, such as mental retardation and hand-foot anomalies (Table 2 and Figure 1).

To favour the search for positional candidate genes, the gained or lost genomic regions should be as small as possible. One example is the $500-\mathrm{kb}$ deletion in $7 \mathrm{p} 21.1$ in our patient 58 : of the two genes 
a

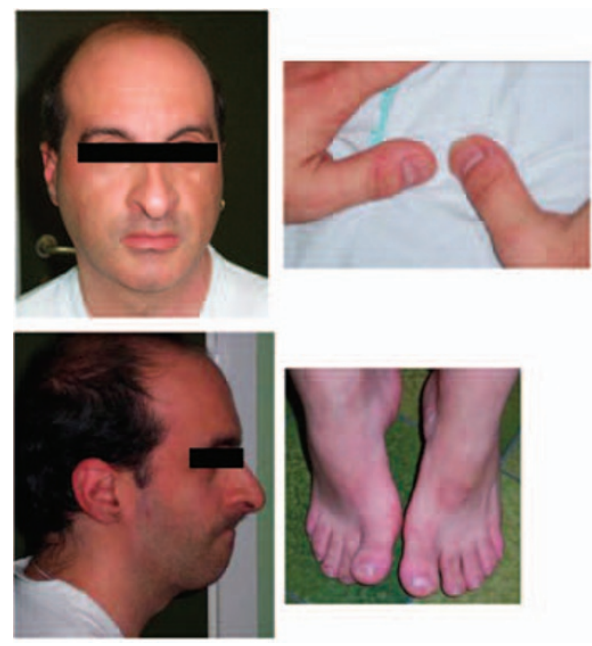

pt 29: dup (17)(q11.2) b

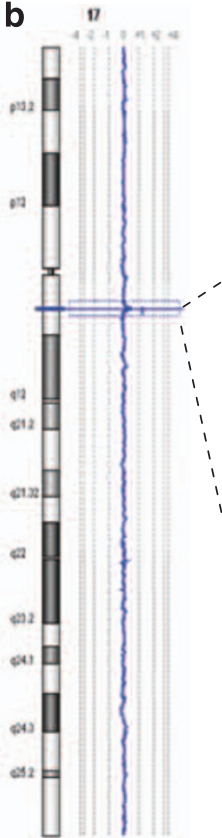

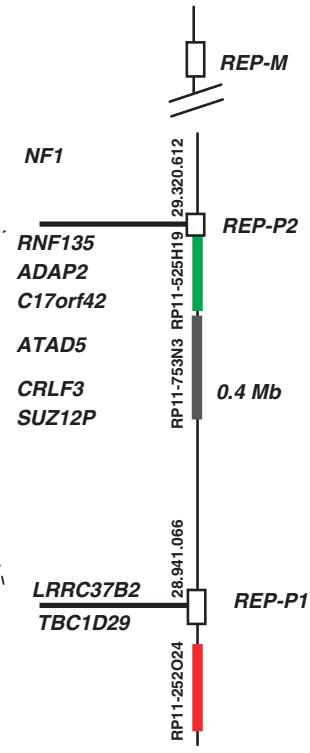
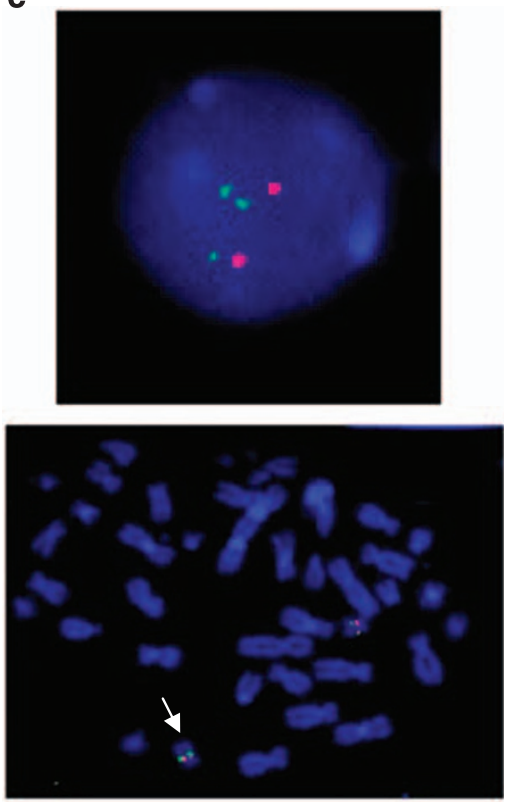

Figure 3 Features of patient 29 (a), aCGH profile (b), and zoom of the region involved in the duplication, with the mapped genes and NF1-duplicons (c).

located in the deleted region, it is difficult to link HDAC9 to the clinical presentation of RSTS as it encodes a factor (histone deacetylase) that has the opposite role of HAT domain of CREBBP (Figure 2d), but TWIST1 may be relevant (Figure 2d) as it encodes a transcription factor implicated in skeletal development and its mutations lead to SCS, a genetic autosomal dominant condition characterised by acrocephalosyndactyly and dysmorphisms. TWIST1 has been suggested as candidate gene for RSTS, ${ }^{10,24}$ on the basis of its nature as a master switch of skeleton morphogenesis and the fact that, like $C R E B B P$, it regulates the development of multiple target genes; however, only a targeted mutational scan of CREBBP- and EP300-negative patients with a clinical diagnosis of RSTS can validate its potential role. The conclusion that can be drawn from the described case is that the overlapping features of SCS and RSTS should be considered when clinically diagnosing the highly different phenotypes of RSTS. As shown in Table 1, patient 58 was given a 'possible' clinical RSTS diagnosis because his phenotype mimics RSTS but also shares some of the signs shown by SCS patients carrying a microdeletion involving the same region or point mutations in the TWIST1 gene (Table 2). This 'borderline' phenotype confirms the difficulty of distinguishing the two syndromes in patients with signs of both.

A question of differential diagnosis is also raised by patient 51 , who carries the large $4.3 \mathrm{Mb} 2 \mathrm{q} 22.3 \mathrm{q} 23.1$ deletion that includes the zincfinger homeobox 1B (ZEB2) gene (Figure 2a) and was initially given a diagnosis of 'probable' RSTS (Table 1). Point mutations of the ZEB2 gene or microdeletions of the region encompassing it lead to MWS, a genetic autosomal dominant syndrome that is characterised by microcephaly, mental retardation and distinct facial features. ${ }^{25}$ It seems that haploinsufficiency of ZEB2 and other genes mapping to the same region may explain the phenotype of a patient who has been diagnosed as having RSTS but also has signs overlapping those of MWS (Table 2).

The genomic rearrangements in the remaining two patients carrying de novo imbalances are too gross to identify probable RSTS candidate genes and do not encompass any key gene associated with a well-defined syndrome (Figure 2).
Patient 14 carries a 9-Mb deletion in 2q24.3q31.1 that involves $>50$ genes, the largest imbalance observed. The very high number of genes precludes any correlation between the haploinsufficiency of specific dose-sensitive genes and the overall phenotype, but some of the master genes mapping to this gene-rich region deserve comment. Differently sized deletions $(5-33 \mathrm{Mb})$ that partially overlap that of patient 14 and include the HOXD gene cluster have been described in patients with distinct phenotypes except for variable limb abnormalities. ${ }^{26-29}$ The HOXD genes are members of a homeobox gene family encoding highly conserved transcription factors that have an important morphogenetic role in all multicell organisms, mainly by acting on the development of the central nervous system, the axial skeleton, the gastrointestinal and urogenital tracts, and limbs, ${ }^{30}$ and they have been suggested as good candidates for causing the limb anomalies in patients with a $2 \mathrm{q} 24 \mathrm{q} 31$ deletion. ${ }^{31}$ Interestingly, FISH mapping of the distal deletion breakpoint of our patient showed that it lies outside the HOXD gene cluster, but includes HOXD13 and the regulatory elements of the whole cluster (Figure $2 b$ ). The absence of the HOXD regulatory elements may explain why the digital anomalies are much milder than those of patients with a deletion including the HOXD cluster, as it has been previously pointed out in the case of a patient carrying a similar deletion except for the additional involvement of SNC cluster genes. ${ }^{32}$

The deletion includes the genes DLX1 and DLX2 (which encode homeobox transcription factors that have a role in controlling craniofacial patterning and the differentiation of inhibitory neurons in the forebrain), and their haploinsufficiency may be responsible for our patient's neurological and dysmorphic phenotype. The deleted region also includes the $A T F 2$ gene which, like CREBBP, ${ }^{33-35}$ encodes a HAT that specifically acetylates histones $\mathrm{H} 2 \mathrm{~B}$ and $\mathrm{H} 4$.

The de novo duplication at $3 \mathrm{p} 13 \mathrm{p} 12.3$ observed in patient 61 encompasses a large $(5 \mathrm{Mb})$ but gene-poor region. Among the involved genes, neither the roundabout, axon guidance receptor, homologue $2(\mathrm{ROBO} 2)^{36}$ nor contactin 3 (CNTN3) seem to be suitable candidates for RSTS. However, the patient's corpus callosum agenesis and autistic traits may be attributable to the dosage alteration 
of the $\mathrm{ROBO} 2$ gene, which may have a role in axon guidance along the dorsoventral axis in the forebrain ${ }^{37}$ and could be related to the autistic phenotype. $^{38}$

The three inherited imbalances may be of interest because they are all private and none of them is included in the databases of polymorphic variants being yet undescribed in the normal population at a frequency $>1 \%$. Their role in the clinical phenotypes of the carrier patients remains unclear, although reduced penetrance may account for the absence of the putatively associated phenotype in the healthy parent.

The $400-\mathrm{kb}$ dup17q11.2 of patient 29 (Figure $3 \mathrm{~b}$ ) is peculiar because it is embedded in the region deleted in Neurofibromatosis type 1 (NF1) microdeletion syndrome and localised between the subduplicons NF1-REP-P1 and REP-P2. REP-P1 mediates the common NF1 microdeletions with the paralogous NF1-REP-M, ${ }^{39}$ whereas REP-P2 has not been reported to be involved in NF1 deletions. This imbalance is the exact reciprocal duplication of a recently reported deletion involving the same region, ${ }^{40}$ both of which can be considered genomic rearrangements insofar as they are mediated by non-allelic homologous recombination (NAHR) of REP-P1 and REP-P2. The deletion described by Douglas et $a l^{40}$ have been associated with an overgrowth syndrome that is presumably because of the haploinsufficiency of the RNF135 gene, which has been found to be mutated in another group of patients with the same overgrowth syndrome. In our case, the duplication is inherited and so it is only possible to hypothesise that the gain of dose of the genes localised in the duplicated interval (RNF135, ADAP2, C17orf42, ATAD5, CRLF3 and $L R R C 37 B 2$ ) (Figure 3c) may contribute to the patient's skeletal anomalies (Figure 3a) and mental retardation. In general, the phenotype resulting from duplications that are even much larger than this is subtle and may be overlooked, as is indicated by the adult age at which a diagnosis was suggested for our patient.

In conclusion, this study is the first systematic array CGH analysis of a cohort of patients with RSTS or RSTS-like features previously screened for mutations in the main CREBBP gene and deletions in regions harbouring the causative $C R E B B P$ and $E P 300$ genes. The large proportion of chromosomal rearrangements in regions other than those of $C R E B B P / E P 300$ genes confirms that array CGH is a suitable diagnostic approach. As no consensus list of diagnostic criteria is yet available for RSTS, array-CGH can be considered a useful means of differentiating patients with overlapping clinical signs. In relation to its use in identifying novel positional RSTS candidate genes, further studies may detect recurrent imbalances or validate/exclude the role of TWIST1 in the aetiology of RSTS.

It is very difficult to interpret the effect of the four de novo chromosome abnormalities found in our patients, and it is not possible to exclude a coincidental finding because of the extremely broad clinical spectrum of RSTS or a cryptic differential diagnosis. Furthermore, the fact that the microdeletions and microduplication of cases 78, 29 and 45 are inherited from healthy parents but not listed as CNVs makes it difficult to interpret their contribution to the clinical phenotype.

\section{CONFLICT OF INTEREST}

The authors declare no conflict of interest.

\section{ACKNOWLEDGEMENTS}

We would like thank the patients' families for participating in this study, and the clinicians who provided some of the patients (Professor G Cocchi, Centro per lo Studio delle Malformazioni Congenite, Istituto Clinico di Pediatria preventiva e Neonatologia, University of Bologna, and Professor
ML Giovannucci-Uzielli, Unità di Genetica, Dip Pediatria, Osp Meyer, University of Florence). This study was supported by 2007-2008 grant from the Associazione Studio Malformazioni (ASM) to LL (University of Milan) and AS (Fondazione Ospedale Maggiore, Policlinico, Regina Elena, Mangiagalli). We would also like to thank the Galliera Genetic Bank Italian Telethon project GTF4003 for providing us with lymphoblastoid cellular lines of some of the studied patients. We gratefully acknowledge our feedback discussion with the group of Dr Angel Barco (Universidad Miguel Hernandez De Elche, Alicante, Spain) in the framework of the Italy-Spain bilateral MIUR project on RSTS (IT08143C4F).

1 Rubinstein JH, Taybi H: Broad thumbs and toes and facial abnormalities. A possible mental retardation syndrome. Am J Dis Child 1963; 105: 588-608.

2 Miller RW, Rubinstein JH: Tumors in Rubinstein-Taybi syndrome. Am J Med Genet 1995; 56: 112-115.

3 Masuno M, Imaizumi K, Ishii T, Kuroki Y, Baba N, Tanaka Y: Pilomatrixomas in Rubinstein-Taybi syndrome. Am J Med Genet 1998; 77: 81-82.

4 Schepis C, Greco D, Siragusa M, Batolo D, Romano C: Rubinstein-Taybi syndrome with epidermal nevus: a case report. Pediatr Dermatol 2001; 18: 34-37.

5 Petrij F, Giles RH, Dauwerse HG et al: Rubinstein-Taybi syndrome caused by mutations in the transcriptional coactivator CBP. Nature 1995; 376: 348-351.

6 Roelfsema JH, White SJ, Ariyürek Y et al: Genetic heterogeneity in Rubinstein-Taybi syndrome: mutations in both the CBP and EP300 genes cause disease. Am J Hum Genet 2005; 76: 572-580.

7 Giordano A, Avantaggiati ML: p300 and CBP: partners for life and death. J Cell Physiol 1999; 181: 218-230.

8 McGaughran JM, Gaunt L, Dore J, Petrij F, Dauwerse HG, Donnai D: Rubinstein-Taybi syndrome with deletions of FISH probe RT1 at 16p13.3: two UK patients. J Med Genet 1996; 33: 82-83.

9 Bartsch 0, Wagner A, Hinkel GK et al: FISH studies in 45 patients with RubinsteinTaybi syndrome: deletions associated with polysplenia, hypoplastic left heart and death in infancy. Eur J Hum Genet 1999; 7: 748-756.

10 Petrij F, Dauwerse HG, Blough RI et al: Diagnostic analysis of the Rubinstein-Taybi syndrome: five cosmids should be used for microdeletion detection and low number of protein truncating mutations. J Med Genet 2000; 37: 168-176.

11 Gervasini C, Castronovo P, Bentivegna A et al: High frequency of mosaic CREBBP deletions in Rubinstein-Taybi syndrome patients and mapping of somatic and germ-line breakpoints. Genomics 2007; 90: 567-573.

12 Coupry I, Monnet L, Attia AA, Taine L, Lacombe D, Arveiler B: Analysis of CBP (CREBBP) gene deletions in Rubinstein-Taybi syndrome patients using real-time quantitative PCR. Hum Mutat 2004; 23: 278-284.

13 Kriek M, Knijnenburg J, White SJ et al: Diagnosis of genetic abnormalities in developmentally delayed patients: a new strategy combining MLPA and array-CGH. Am J Med Genet A 2007; 143: 610-614.

14 Udaka T, Kurosawa K, Izumi $\mathrm{K}$ et al: Screening for partial deletions in the CREBBP gene in Rubinstein-Taybi syndrome patients using multiplex PCR/liquid chromatography. Genet Test 2006; 10: 265-271.

15 Bartsch O, Schmidt S, Richter M et al: DNA sequencing of CREBBP demonstrates mutations in $56 \%$ of patients with Rubinstein-Taybi syndrome (RSTS) and in another patient with incomplete RSTS. Hum Genet 2005; 117: 485-493.

16 Bentivegna A, Milani D, Gervasini $C$ et al: Rubinstein-Taybi syndrome: spectrum of CREBBP mutations in Italian patients. BMC Med Genet 2006; 7: 77.

17 Roelfsema JH, Peters DJ: Rubinstein-Taybi syndrome: clinical and molecular overview. Expert Rev Mol Med 2007; 9: 1-16.

18 Bartholdi D, Roelfsema JH, Papadia F et al: Genetic heterogeneity in Rubinstein-Taybi syndrome: delineation of the phenotype of the first patients carrying mutations in EP300. J Med Genet 2007; 44: 327-333.

19 Zimmermann N, Acosta AM, Kohlhase J, Bartsch O: Confirmation of EP300 gene mutations as a rare cause of Rubinstein-Taybi syndrome. Eur J Hum Genet 2007; 15: 837-842.

20 Foley P, Bunyan D, Stratton J, Dillon M, Lynch SA: Further case of Rubinstein-Taybi syndrome due to a deletion in EP300. Am J Med Genet A 2009; 149A: 997-1000.

21 Stef M, Simon D, Burgelin I et al: Testing and improving experimental parameters for the use of low molecular weight targets in array-CGH experiments. Hum Mutat 2006; 27: 1143-1150.

22 Lichter $\mathrm{P}$, Cremer T: Chromosome analysis by non-isotopic in situ hybridization; in Rooney DE, Czepulkowski BH (eds):: Human Cytogenetics: A Practical Approach, 2nd edn. Oxford: IRL, 1992, Vol 1, pp 157-192.

23 Coupry I, Roudaut $\mathrm{C}$, Stef $\mathrm{M}$ et al: Molecular analysis of the CBP gene in 60 patients with Rubinstein-Taybi syndrome. J Med Genet 2002; 39: 415-421.

24 Lowry RB: Overlap between Rubinstein-Taybi and Saethre-Chotzen syndromes: a case report. Am J Med Genet Supp/ 1990; 6: 73-76.

25 Garavelli L, Zollino M, Mainardi PC et al: Mowat-Wilson syndrome: facial phenotype changing with age: study of 19 Italian patients and review of the literature. Am J Med Genet A 2009; 149A: 417-426.

26 Nixon J, Oldridge M, Wilkie AO, Smith K: Interstitial deletion of $2 q$ associated with craniosynostosis, ocular coloboma, and limb abnormalities: cytogenetic and molecular investigation. Am J Med Genet 1997; 70: 324-327. 
27 Slavotinek A, Schwarz C, Getty JF, Stecko O, Goodman F, Kingston H: Two cases with interstitial deletions of chromosome 2 and sex reversal in one. Am J Med Genet 1999; 86: 75-81.

28 Bijlsma EK, Knegt AC, Bilardo CM et al: Increased nuchal translucency and split-hand/ foot malformation in a fetus with an interstitial deletion of chromosome $2 \mathrm{q}$ that removes the SHFM5 locus. Prenat Diagn 2005; 25: 39-44.

29 Svensson AM, Curry CJ, South ST et al: Detection of a de novo interstitial $2 q$ microdeletion by CGH microarray analysis in a patient with limb malformations, microcephaly and mental retardation. Am J Med Genet A 2007; 143A: 1348-1353.

30 Goodman FR: Limb malformations and the human HOX genes. Am J Med Genet 2002; 112: 256-265.

31 Del Campo M, Jones MC, Veraksa AN et al: Monodactylous limbs and abnormal genitalia are associated with hemizygosity for the human $2 \mathrm{q} 31$ region that includes the HOXD cluster. Am J Hum Genet 1999; 65: 104-110.

32 Pescucci C, Caselli R, Grosso S et al: 2q24-q31 deletion: report of a case and review of the literature. Eur J Med Genet 2007; 50: 21-32.

33 Ogryzko VV, Schiltz RL, Russanova V, Howard BH, Nakatani Y: The transcriptional coactivators p300 and CBP are histone acetyltransferases. Cell 1996; 87: 953-959.
34 Schiltz RL, Mizzen CA, Vassilev A, Cook RG, Allis CD, Nakatani Y: Overlapping but distinct patterns of histone acetylation by the human coactivators p300 and PCAF within nucleosomal substrates. J Biol Chem 1999; 274: 1189-1192.

35 Kalkhoven E: CBP and p300: HATs for different occasions. Biochem Pharmacol 2004; 68: 1145-1155.

$36 \mathrm{Lu} \mathrm{W}$, van Eerde AM, Fan X et al: Disruption of ROBO2 is associated with urinary tract anomalies and confers risk of vesicoureteral reflux. Am J Hum Genet 2007; 80: 616-632.

37 Devine CA, Key B: Robo-Slit interactions regulate longitudinal axon pathfinding in the embryonic vertebrate brain. Dev Biol 2008; 313: 371-383.

38 Anitha A, Nakamura K, Yamada K et al: Genetic analyses of roundabout (ROBO) axon guidance receptors in autism. Am J Med Genet B Neuropsychiatr Genet 2008; 147B: 1019-1027.

39 Dorschner MO, Sybert VP, Weaver M, Pletcher BA, Stephens K: NF1 microdeletion breakpoints are clustered at flanking repetitive sequences. Hum Mol Genet 2000; 9: 35-46.

40 Douglas J, Cilliers D, Coleman K et al: Mutations in RNF135, a gene within the NF1 microdeletion region, cause phenotypic abnormalities including overgrowth. Nat Genet 2007; 39: 963-965. 